\title{
OBJECT-ORIENTED CHANGE DETECTION BASED ON SPATIOTEMPORAL RELATIONSHIP IN MULTITEMPORAL REMOTE-SENSING IMAGES
}

\author{
Liang $\mathrm{Li}^{\mathrm{a}, \mathrm{b}, *}$, Guowei Ying ${ }^{\mathrm{b}}$, Xuehu Wen ${ }^{\mathrm{b}}$, Yun Zhang ${ }^{\mathrm{b}}$ \\ ${ }^{a}$ The Third Academy of Engineering of Surveying and Mapping, 2 Xinjun Road, 610500 Chengdu, China \\ ${ }^{\mathrm{b}}$ Geographic National Condition Monitoring Engineering Research Center of Sichuan Province, 2 Xinjun \\ Road,610500 Chengdu, China-liliang1987wuda@163.com,331293996@qq.com,550208129@qq.com, \\ zzzyun@vip.sina.com
}

Commission $\times \times$, WG $\times \times$

KEY WORDS: object-oriented, change detection, post classification comparison (PCC), spatiotemporal relationship, Markov Random Field (MRF), remote-sensing images

\begin{abstract}
:
In this paper a novel object-oriented change detection approach in multitemporal remote-sensing images is proposed. In order to improve post classification comparison (PCC) performance, we propose to exploit spatiotemporal relationship between two images acquired at two different times. The probabilities of class transitions are used to describe the temporal dependence information, while the Markov Random Field (MRF) model is utilized to represent the spatial-contextual information. Training sets are required to get initial classification results by maximum likelihood method (ML).Then an estimation procedure: iterated conditional mode (ICM) is present to revise the classification results. Change detection (change/no change) and change type recognitions (from-to types of change) are achieved by compare classification maps acquired at two different times. Experimental results on two QuickBird images confirm that the proposed method can provide higher accuracy than the PCC method, which ignores spatiotemporal relationship between two images.
\end{abstract}

\section{INTRODUCTION}

Remote-Sensing images with large cover area and short revisit time are widely used in land use/land cover change detection since its birth. Land use/land cover change detection using remote sensing technology has always been an important research topic in remote sensing applications [1]. According to the basic unit of the land use/land cover changes, change detection methods can be cataloged into two types: pixel-based method and object-oriented method.

Many change detection algorithms belong to the former approach [2]-[10]. The most popular methods of them are the image differencing algorithm [3] [5],change vector analysis [6]-[10] and postclassification comparison[11].The image differencing algorithm is an unsupervised technique as well as change vector analysis, while PCC is a supervised technique for change detection. The image differencing algorithm is usually divided into three steps:1) pre-processing. The two images should be coregistered so that pixels with the same coordinates are corresponding to the same area on the ground.2) Difference image generation. The two images are subtracted, pixel by pixel, to generate a further image difference image. 3) Threshold decision. Under the assumption of few changes between two different images, changes are located in the tails of probability density function of the difference image. They can be detected through a certain threshold gained by ostu algorithm or bayesian theory. Change vector analysis can not only detect changes but also provide "from-to" types of change information using the gray

\footnotetext{
* Corresponding author: Liang Li (1987-), male, PhD candidate, majors in intelligent interpretation of remote sensing imagery.E-mail: liliang1987wuda@163.com
}

value of all bands. The gray values corresponding with different bands of a pixel at each time compose a vector. Then for each couple of pixels, change vector is computed by subtracting vectors acquired at the two times. The changes in land-cover classes are determined based on both the magnitude and the direction of the change vector [6]. The limitation of image differencing algorithm and change vector analysis is the false alarm caused by difference in light conditions, atmospheric conditions, and sensor calibration at the two acquisition images concerned. In addition, the acquisition of the optimal threshold value is also a bottleneck. However, PCC approach can overcome these problems. Two images are classified separately according to the training sets. It performs change detection and change type recognitions by comparing the classification maps between two images. The accuracy of this method depends on the performances of the classification maps. Therefore, training sets play an important part in this technique. Typical training sets are required.

With the development of remote sensing sensors, the spatial resolution of remote sensing images has been greatly improved. Therefore, abundant spectral information and texture information are contained in high-resolution imagery. Pixel-based technique for change detection becomes ineffective in highresolution imagery. This is due to the fact that pixelbased technique doesn't take into account the spatialcontextual information in the multitemporal images considered. The object-oriented method is more suitable for high-resolution imagery [12]-[16]. Objects are composed of a set of pixels which are adjacent in space and spectrally similar to each other. Thus object which contains various kind of information such as spatial, spectral and shape information, can be regarded as a homogeneous entirety. Image segmentation and GIS data are two ways to acquire the objects. The basic unit of object-oriented method is an object. In this way, it can suppress the salt and pepper noise 
effectively in high-resolution imagery. Some objectoriented methods have been developed for change detection, such as the multilevel model [12], Kolmogorov-Simirnov test technique [13], OCI method [14], post-classification comparison [15]. Although the use of object-oriented postclassification comparison is widespread because it can detect changes and provide from-to types of change,it exhibits two major drawbacks: lack of temporal dependence information between the two images of the same area acquired at the two times and lack of spatial-contextual information in single image.

In order to overcome these problems, object- oriented PCC technique considering spatiotemporal relationship is proposed in this paper. First, image segmentation with two images using marker-based watershed algorithm is applied to obtain image objects. According to acquired training sets, object-oriented classification using ML method for each image are performed to get initial classification maps. Next, spatiotemporal relationship which involves both temporal dependence information and spatialcontextual information, is presented to revise compound classification maps. Finally, change detection and change type recognitions are achieved by comparing the revised two classification maps. The processing flow of the proposed method is shown in Figure 1.

This paper is organized into five sections.In Section 1, Marker-based watershed image segmentation is described. Object-oriented PCC technique considering spatiotemporal relationship is proposed in Section 2. Experimental results and discussions are reported in Section 3. The conclusion is drawn in Section 4.

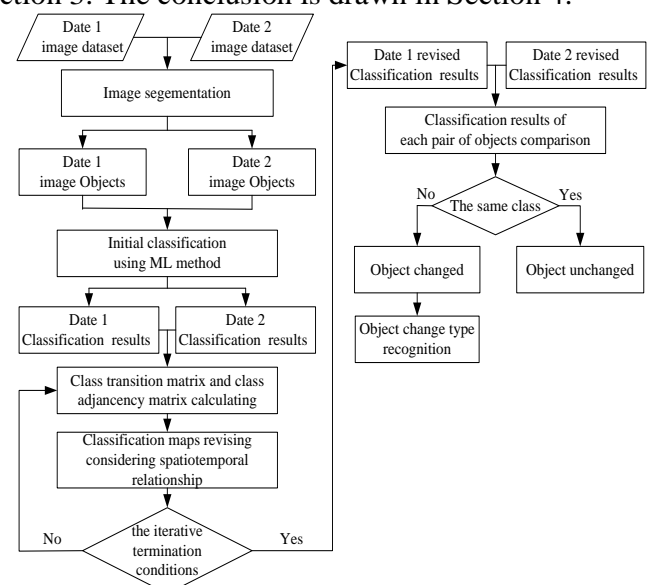

Figure 1. Schematic representation of the proposed technique

\section{MARKER-BASED WATERSHED IMAGE SEGMENTATION}

In the proposed method, image segmentation is employed to gain image objects. The definition of image segmentation is detailed in the following. Let $R$ be the image for segmentation, and let $H$ be a homogeneity measure in the feature space. The segmentation map is a partition $P(X)$ composed of $N$ disjoint regions $R_{i}$ with $R_{i} \subseteq R, i=1,2, \ldots, N$. The regions should satisfy the following conditions:

(1) $\bigcup_{i=1}^{N} R_{i}=R, i=1,2, \ldots, N$.
(2) $R_{i} \cap R_{j}=\varnothing$ for $i \neq j$, where $i, j=1,2, \ldots, N$.

(3) $H\left(R_{i}\right)=$ true $, i=1,2, \ldots, N$.

(4) $H\left(R_{i} \cup R_{j}\right)=$ false, where $R_{i}$ and $R_{j}$ are adjacent, $i, j=1,2, \ldots, N$.

(5) Pixels in $R_{i}$ are connected, $i=1,2, \ldots, N$.

Conditions (1) and (5) ensure that the image is divided into $N$ connected regions. Condition (2) guarantees that $N$ connected regions are non-overlapping regions. Condition (3) determines the homogeneity of the region, and condition (4) assures the heterogeneity between the region and its adjacent region.

Many segmentation strategies have been proposed in recent years (e.g., region growing method, edge-based technique, etc.) [17]-[21].In this paper, we adopt a marker-based watershed algorithm to segment the image which can reduce over-segmentation in traditional watershed algorithm. Marks are flat regions composed of a set of pixels with low gradient values. Each mark indicates the presence of an object. There are three main steps in the marker-based watershed approach. These steps are detailed in the following.

1) Extraction of marker image. Each pixel corresponds to a certain threshold value. Marks are composed of pixels whose gradient values are less than the threshold value. The marks in the image are set to 1 , and the others are set to 0 .This produces a marked binary image.

2) Elimination of false markers. Image noise can produce false marks which can result in oversegmentation. False marks can be discarded adopting minimum size constraint.

3) Segmentation by marker-based watershed algorithm.Meyer proposed a direct marker-based watershed algorithm by immersion, depending on the priority queues [22],[23].The higher priority of a pixel is, the lower is the gradient value. This algorithm can be summarized as follows: (a) labeling the markers and initialization of the priority queues, (b) region growing. A pixel $p$ is extracted from the end of the highest priority queue. The neighbor pixels of $p$ which are still outside the queue are put into the queue according to the gradient value, however, when the gradient value is lower than the priority of $p$, this neighbor is put into the queue with the same priority of pixel $p$. If pixel $p$ has in its neighborhood one and only one labeled region, it is aggregated to this region and if pixel $p$ is a neighbor of two regions with different labels, $p$ is a boundary pixel and gets a special label.

For a more detailed description of the marker-based watershed approach we refer the readers to [22].

In order to get a segmentation map shared by two considered images, we generate a new image by combining two multispectral images. The bands of new image are composed of bands from two images. Marker-based watershed image segmentation technique for the new image is adopted.

\section{OBJECT-ORIENTED CHANGE DETE- CTION TECHNIQUE BY COMPOUND CLASSIFICATION CONSIDERING SPAT- IOTEMPORAL RELATIONSHIP}

Let us consider two co-registered multispectral images, acquired at two different times $t_{1}$ and $t_{2}$ with the 
same area on the ground. Two images are divided into $N$ disjoint objects after image segmentation. Let $X_{1}$ and $X_{2}$ be $d$-dimensional feature vectors at time $t_{1}$ and $t_{2}$,where $X_{1}=\left\{X_{1}(k), k=1,2, \ldots, N\right\}$ and $X_{2}=\left\{X_{2}(k), k=1,2, \ldots, N\right\}$, where $X_{i}(k)$ indicates the feature vector of $k$-th object at the time $t_{i}, i=1,2$. Let $\Omega=\left\{\omega_{1}, \omega_{2}, \ldots, \omega_{m}\right\}$ be the set of $m$ land-cover classes at time $t_{1}$, and $\Phi=\left\{v_{1}, v_{2}, \ldots, v_{n}\right\}$ be the set of $n$ land-cover classes at time $t_{2}$.The classification results of each image can be regarded as a random experiment. Let the set $C=\left\{C_{p}, 1 \leq p \leq L\right\}$, with $L=m^{N}$, be composed of all the possible sets of classification results in the image at $t_{1}$, where $C_{p}=\left\{C_{p}(k), k=1,2, \ldots, N\right\}$, with $C_{p}(k) \in \Omega . C_{p}(k)$ indicates the classification result of $k$-th object in $C_{p}$ which denotes the results of $p$-th random experiment at $t_{1}$. Let the set $T=\left\{T_{q}, 1 \leq q \leq S\right\}$, with $S=n^{N}$, be composed of all the possible sets of classification results in the image at $t_{2}$, where $T_{q}=\left\{T_{q}(k), k=1,2, \ldots, N\right\}$, with $T_{q}(k) \in \Phi$. $T_{q}(k)$ indicates the classification result of $k$-th object in $T_{q}$ which denotes the results of $q$-th random experiment at $t_{2}$. Last, let us define the neighbor system of the $k$-th object as $\varepsilon(k)=\{i$, object $i$ and $k$ are adjacent, $1 \leq i \leq N\}$.

According to the Bayesian formulation, the optimal sets of classification results $C_{t}$ and $T_{h}$ that minimize the classification error can be derived by using the following decision rule:

$$
\left(C_{t}, T_{h}\right)=\underset{C_{p} \in C, T_{q} \in T}{\arg \max }\left\{P\left(C_{p}, T_{q}\right) /\left(X_{1}, X_{2}\right)\right\}
$$

The couple of classification results $\left(C_{t}, T_{h}\right)$ that provides the maximum in (1) also provides the following maxima:

$$
\begin{aligned}
& \max _{C_{p} \in C, T_{q} \in T}\left\{P\left(C_{p}, T_{q}\right) p\left[\left(X_{1}, X_{2}\right) /\left(C_{p}, T_{q}\right)\right] / P\left(X_{1}, X_{2}\right)\right\} \\
& \Leftrightarrow \max _{C_{p} \in C, T_{q} \in T}\left\{P\left(C_{p}, T_{q}\right) p\left[\left(X_{1}, X_{2}\right) /\left(C_{p}, T_{q}\right)\right]\right\}
\end{aligned}
$$

where $P\left(C_{p}, T_{q}\right)$ is a priori probability for the classification results $C_{p}$ and $T_{q}, p\left[\left(X_{1}, X_{2}\right) /\left(C_{p}, T_{q}\right)\right]$ is the conditional density function of the feature vectors in the images $X_{1}$ and $X_{2}$, given the classes $C_{p}$ and $T_{q}$, and $P\left(X_{1}, X_{2}\right)$ can be discarded since it is independent of $C_{p}$ and $T_{q}$.

Formula (2) is equivalent to the formula (3).

$$
\max _{C_{p} \in C, T_{q} \in T}\left\{P\left(T_{q} / C_{p}\right) P\left(C_{p}\right) p\left[\left(X_{1}, X_{2}\right) /\left(C_{p}, T_{q}\right)\right]\right\}
$$

where $P\left(T_{q} / C_{p}\right)$ is the probability of $T_{q}$, given the classification results $C_{p}$, and $P\left(C_{p}\right)$ is a priori probability for the classification results $C_{p}$.In order to simplify the problem, we make two assumptions. 1)The feature vectors $X_{1}$ only depends on the classification results $C_{p}$, and the feature vectors $X_{2}$ only depends on the classification results $T_{q}$. Thus the following equation is expressed:

$$
p\left[\left(X_{1}, X_{2}\right) /\left(C_{p}, T_{q}\right)\right]=p\left(X_{1} / C_{p}\right) p\left(X_{2} / T_{q}\right)
$$
where $p\left(X_{1} / C_{p}\right)$ is the conditional probability of feature vectors $X_{1}$, given the classification results $C_{p}$ and $p\left(X_{2} / T_{q}\right)$ is the conditional probability of feature vectors $X_{2}$,given the classification results $T_{q}$.

2)The following conditional independence are assumed:

$$
\begin{aligned}
& P\left(T_{q} / C_{p}\right)=\prod_{k=1}^{N} P\left(T_{q}(k) / C_{p}(k)\right) \\
& p\left(X_{1} / C_{p}\right)=\prod_{k=1}^{N} p\left(X_{1}(k) / C_{p}(k)\right) \\
& p\left(X_{2} / T_{q}\right)=\prod_{k=1}^{N} p\left(X_{2}(k) / T_{q}(k)\right)
\end{aligned}
$$

where $P\left(T_{q}(k) / C_{p}(k)\right) \quad$ is the transition probability from land-cover class $C_{p}(k)$ at $t_{1}$ to $T_{q}(k) \quad$ at time $t_{2} \cdot p\left(X_{1}(k) / C_{p}(k)\right)$ and $p\left(X_{2}(k) / T_{q}(k)\right)$ are the conditional probabilities at the two times.

The assumption analysis to that defined by (4) was introduced in reference [11].It is worth noting that the effectiveness of assumption (2) is difficult to deduce in theory. The formulas (5)-(7) are approximate formulas and more critical than the others. However, researchers have been applied the formula (6), (7) to change detection application [4] [11].It can be regarded as a good trade-off between computational efficiency and estimation accuracy.

By substituting (4)-(7) into (3), we can obtain following formula:

$\max _{C_{p} \in C, T_{q} \in T}\left\{P\left(C_{p}\right) \prod_{k=1}^{N}\left[P\left(T_{q}(k) / C_{p}(k)\right) p\left(X_{1}(k) / C_{p}(k)\right) p\left(X_{2}(k) / T_{q}(k)\right)\right]\right\}$

\subsection{Markov Random Field}

$P\left(C_{p}\right)$ is a prior probabilities of the classification results for all objects in the image acquired at time $t_{1}$. The objects involved in the image at $t_{1}$ are not spatially independent. The object is likely to be surrounded by objects with the same class, for example, the lakes prefer to adjacent to the lakes rather than the buildings. MRF technique is used to model the spatial-contextual information between objects [23]. $P\left(C_{p}\right)$ can be expressed as:

$$
\begin{aligned}
& P\left(C_{p}\right)=\prod_{k=1}^{N} p\left(C_{p}(k) /\left\{C_{p}(j), j \neq k, j, k=1,2, \ldots, N\right\}\right) \\
= & \prod_{k=1}^{N} p\left(C_{p}(k) /\left\{C_{p}(j), j \in \varepsilon(k)\right\}\right) \\
= & \prod_{k=1}^{N} \frac{1}{Z} \exp \left[-U\left(C_{p}(k) /\left\{C_{p}(j), j \in \varepsilon(k)\right\}\right)\right]
\end{aligned}
$$


where $U($.$) is the Gibbs energy function and \mathrm{Z}$ is a normalized constant. $U\left(C_{p}(k) /\left\{C_{p}(j), j \in \varepsilon(k)\right\}\right)$ is defined as:

$U\left(C_{p}(k) /\left\{C_{p}(j), j \in \varepsilon(k)\right\}\right)=\sum_{j \in \varepsilon(k)} \beta \sigma\left(C_{p}(k), C_{p}(j)\right)$

where $\sigma$ is potential function and $\beta$ is the weight of spatial-contextual information in the process. The higher is the adjacent probability of the two objects, the smaller is the $\sigma$ function value. $\sigma$ is expressed as :

$$
\sigma\left(C_{p}(k), C_{p}(j)\right)=-P\left(C_{p}(k) / C_{p}(j)\right)
$$

where $P\left(C_{p}(k) / C_{p}(j)\right)$ is a conditional probability of land-cover class $C_{p}(k)$, given the adjacent class $C_{p}(j)$.

By applying (9)-(11) into (8) and discarding the constant, the following formula can obtain:

$$
\begin{aligned}
\underset{C_{p} \in C, T_{q} \in T}{\max } & \prod_{k=1}^{N}\left\{p\left(X_{1}(k) \mid C_{p}(k)\right) p\left(X_{2}(k) \mid T_{q}(k)\right)\right. \\
& \left.P\left(T_{q}(k) \mid C_{p}(k)\right) \exp \left[\beta \sum_{j \in \varepsilon(k)} P\left(C_{p}(k) / C_{p}(j)\right)\right]\right\}
\end{aligned}
$$

We can transform (12) into (13) using log function.

$$
\begin{aligned}
\max _{C_{p} \in C, T_{q} \in T} & \sum_{k=1}^{N}\left\{\ln p\left(X_{1}(k) \mid C_{p}(k)\right)+\ln p\left(X_{2}(k) \mid T_{q}(k)\right)\right. \\
& \left.+\ln P\left(T_{q}(k) \mid C_{p}(k)\right)+\beta \sum_{j \in \varepsilon(k)} P\left(C_{p}(k) / C_{p}(j)\right)\right\}
\end{aligned}
$$

According to (13),we need to estimate the conditional probabilities $p\left(X_{1}(k) \mid C_{p}(k)\right)$ and $p\left(X_{2}(k) \mid T_{q}(k)\right)$ at the two times, and land-cover transition probability $P\left(T_{q}(k) \mid C_{p}(k)\right)$ which represents the temporal dependence information between the time $t_{1}$ and $t_{2}$, and the probability of land-cover adjacency probability $P\left(C_{p}(k) / C_{p}(j)\right)$ which describes the spatial-contextual information at time $t_{1}$.

\subsection{Conditional Probabilities}

The conditional probabilities $p\left(X_{2}(k) \mid T_{q}(k)\right)$ and $p\left(X_{1}(k) \mid C_{p}(k)\right)$ can be estimated from the training sets of two images. In the Gaussian case, the mean vector $\mu$ and the covariance matrix $\Sigma$ are used to estimate the conditional probabilities. The parameters $\mu$ and $\Sigma$ of each land-cover class can abstract from corresponding training sets.

\subsection{Land-cover class transition and adjacency matrixes}

The probabilities of land-cover class transitions which represent the temporal dependence information between two times establish the following $m \times n$ matrix.

$$
T=\left(\begin{array}{ccccc}
P\left(v_{1} / \omega_{1}\right) & \ldots & P\left(v_{j} / \omega_{1}\right) & \ldots & P\left(v_{n} / \omega_{1}\right) \\
\ldots & \ldots & \ldots & \ldots & \ldots \\
P\left(v_{1} / \omega_{i}\right) & \ldots & P\left(v_{j} / \omega_{i}\right) & \ldots & P\left(v_{n} / \omega_{i}\right) \\
\ldots & \ldots & \ldots & \ldots & \ldots \\
P\left(v_{1} / \omega_{m}\right) & \ldots & P\left(v_{j} / \omega_{m}\right) & \ldots & P\left(v_{n} / \omega_{m}\right)
\end{array}\right)
$$

where the element $P\left(v_{j} / \omega_{i}\right)$ with row $i$ and column $j$ in the matrix denotes the probability of an object belongs to land-cover class $v_{j}$ at time $t_{2}$, on the condition that it belongs to land-cover class $\omega_{i}$ at time $t_{1}$.

The conditional probabilities of the land-cover class adjacency which represent the spatial-contextual information at the time $t_{1}$ compose the following $m \times m$ matrix.

$$
A=\left(\begin{array}{ccccc}
P\left(\omega_{1} / \omega_{1}\right) & \ldots & P\left(\omega_{j} / \omega_{1}\right) & \ldots & P\left(\omega_{m} / \omega_{1}\right) \\
\ldots & \ldots & \ldots & \ldots & \ldots \\
P\left(\omega_{1} / \omega_{i}\right) & \ldots & P\left(\omega_{j} / \omega_{i}\right) & \ldots & P\left(\omega_{m} / \omega_{i}\right) \\
\ldots & \ldots & \ldots & \ldots & \ldots \\
P\left(\omega_{1} / \omega_{m}\right) & \ldots & P\left(\omega_{j} / \omega_{m}\right) & \ldots & P\left(\omega_{m} / \omega_{m}\right)
\end{array}\right)
$$

where the element $P\left(\omega_{j} / \omega_{i}\right)$ with row $i$ and column $j$ in the matrix represents the probability of an object belongs to land-cover class $\omega_{j}$ at time $t_{1}$, on the condition that it is adjacent to the land-cover class $\omega_{i}$ at the same time.

In order to estimate all the probabilities in matrix $T$ and $A$, training sets are required. The training sets should satisfy the following conditions:1)training sets should correspond to the same area on the ground at the two times;2)training sets should contain all the probabilities of the land-cover class transitions and adjacencies [11]. In practical terms, it's impossible to select the training sets to satisfy above mentioned conditions.

Different places at different times with different land policies formulated by local government leads to different matrix $T$ and $A$, for example, in the period of the urbanization and city constructions, the probability of farmland transferred to the building is higher than it transferred to the lake. As a result, historical dataset can't be used to get current land-cover class transition and adjacency matrixes accurately.

Consequently, we estimate the probabilities in matrix $T$ and $A$ from the whole image.

$$
\begin{aligned}
& P\left(v_{j} / \omega_{i}\right)=f\left(\omega_{i}, v_{j}\right) / f\left(\omega_{i}\right) \\
& P\left(\omega_{j} / \omega_{i}\right)=g(\omega, \omega) g \quad \phi
\end{aligned}
$$

where $f\left(\omega_{i}, v_{j}\right)$ is the number of objects with land-cover class $\omega_{i}$ at $t_{1}$ and $v_{j}$ at $t_{2}, g\left(\omega_{i}, \omega_{j}\right)$ is the number of object pairs with land-cover class $\omega_{i}$ and $\omega_{j}$ at $t_{1}, f\left(\omega_{i}\right)$ is the number of objects with land-cover class $\omega_{i}$ at $t_{1}$, and $g\left(\omega_{i}\right)$ is the number of objects adjacent to the object with land-cover class $\omega_{i}$ at $t_{1}$.

\subsection{Iterated conditional mode for compound} classification

The number of all possible classification results at the two times is $(m n)^{N}$. It's impossible to find optimal classification results which verify (13) using exhaustive method since $(m n)^{N}$ is enormous. We propose a fast method by iterated conditional mode 
(ICM) technique [25]. According to ICM, the land-cover classes of the current couple of objects at the two times is determined by the land-cover classes of the other couples of objects and the feature vectors of all couples of objects. ICM technique is realized according to the following steps.

1) Initial classifications of the two images using ML approach independently.

2) Compute the land-cover class transitions and adjacencies matrixes depend on the initial classifications.

3) Update the classification results of the two images which verify (13), for all couples of objects at two times.

4) Repeat 2)-3) until the convergence.

\subsection{Change detection and change type recognition}

The optimal compound classification results $C_{t}$ and $T_{h}$ at the two times can be obtained using aforementioned technique. $C_{t}(k)$ and $T_{h}(k)$ are the classification results of $k$-th object at the two times. Change detection and change type recognition are implemented by comparing $C_{t}(k)$ with $T_{h}(k)$.If $C_{t}(k)$ is not the same land-cover class as $T_{h}(k)$, the object has been changed from $C_{t}(k)$ to $T_{h}(k)$, otherwise it has no change.

\section{EXPERIMENTAL RESULTS AND DISCUSSIONS}

\subsection{Data set description}

The study area is located in the Wuhan city in the center of China. Two images with the same area of $758 \times 623$ pixels are acquired by QuickBird satellite in 2002(time $t_{1}$ ) and 2005(time $t_{2}$ ). The QuickBird imagery is made of four multispectral bands with a spatial resolution of $2.44 \mathrm{~m}$ : the red band, the green band, the blue band, and the near infrared band and one panchromatic band with a spatial resolution of $0.61 \mathrm{~m}$. The bands selected for change detection are four multispectral bands with a spatial resolution of $2 m$ after resampling. The dataset used in the experiments is shown in Figure 2 .

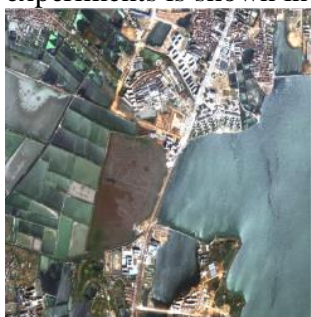

(a)

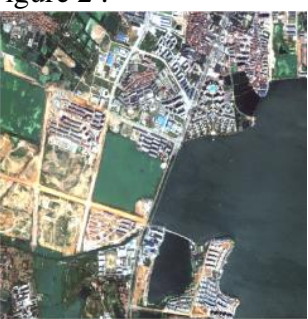

(b)
Figure 2. Change detection imagery acquired in (a) 2002 and (b) $2005(758 \times 623$ pixels, with the spatial resolution of $2 m$ ).

The number of objects extracted from image segmentation by marker-based watershed method is 625 . Figure 3 shows the image segmentation results.

\subsection{Classification results}

There are four classes in the image acquired in 2002 and five classes in the image acquired in 2005. Therefore we selected four classes: lake, building,

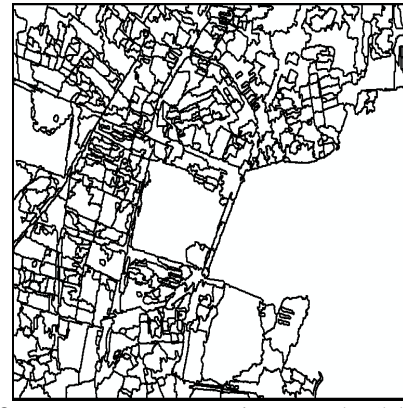

Figure 3. Image segmentation results(black lines denotes the boundary of the objects).

road, farmland at time $t_{1}$ and five classes: lake, building, road, farmland, bare land at time $t_{2}$. Because of the complexity of the remote sensing imagery, subclasses with different spectral information exist in one land-cover class, i.e., the lake contains many subclasses with different sediment concentration and chlorophyll content. This problem will reduce the accuracy of the image classification. In order to solve this problem, training sets containing all subclasses should be selected.

Figure 4 shows the classification results at the two times. The object belongs to the lake on the ground truth involved in red rectangle A is classified to the building in Figure 4(a) using ML method, while it is classified to the lake in Figure 4(c) using the proposed approach. The reason is that this object has similar spectral features as the building, but it's more likely to belong to the lake, considering the spatial-contextual information that the object adjacent to it belongs to the lake.

Many objects belong to bare-land on the ground truth involved in red rectangle B of Figure 4(b) are mistakenly classified as the road since the road and bare-land both have strong reflectivity. However, these objects are classified correctly in Figure $4(d)$ because the objects belong to the lake at time $t_{l}$ are more likely to the lake at time $t_{1}$ are more likely to transform into buildings than the road considering temporal dependence.

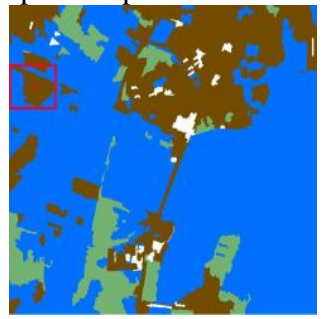

(a)

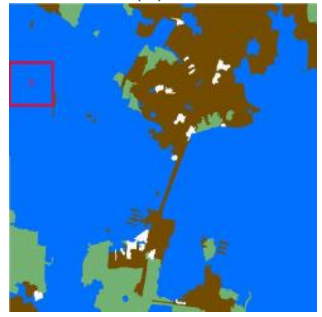

(c)

lake $\square$ building

$\square$ bare land

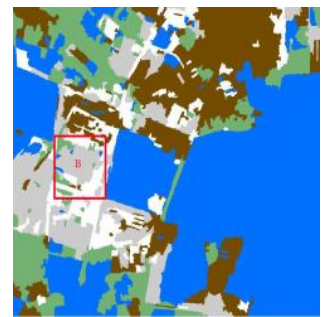

(b)

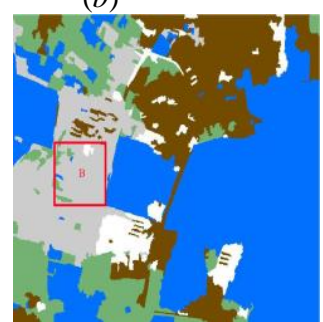

(d)
Figure 4. Classification results: (a) classification maps in the imagery acquired in 2002 using ML method;(b) classification maps in the imagery acquired in 2005 
using ML method;(c) classification maps in the imagery acquired in 2002 using the proposed approach.(d) classification maps in the imagery acquired in 2005 using the proposed approach.

The land-cover class transition and adjacency matrixes after four iterations are shown in Table 1 and 2. The land cover at time $t_{1}$ and $t_{2}$ are given by the columns and rows in Table 1 . The land cover class and the adjacent class at time $t_{1}$ are given by the columns and rows in Table 2.It is easy to observe in Table 1 that the lake tends to transform to other class while the building seldom converts to other class between two different times. Table 2 shows that objects have aggregation effect in space which indicates that objects are more likely adjacent to objects with the same land-cover class, for example, the probability that an object belonging to lake under the condition that the adjacent object belongs to the lake is as high as 0.72 . However the probability that an object belonging to building under the condition that the adjacent object belongs to the road is 0.68 which is very high. The reason is that the buildings usually lie on both sides of the road in China.

The error matrixes of land-cover transitions for PCC method and the proposed method were computed in Table 3 and Table 4. Land-cover transitions from the ground truth area are given in the rows in two tables, while the land-cover transitions detected by pcc method are given in the columns in Table 3 and the land-cover transitions detected by the proposed method are given in the columns in Table 4 ( $i j$ denotes the object transform from $\omega_{i}$ at $t_{1}$ to $v_{j}$ at $\left.t_{2}, i=1,2,3,4, j=1,2,3,4,5\right)$.
Compared the two tables, we can see that the proposed approach can provide better accuracy than PCC method. In particular, the accuracy of 15(lake-bare land) and 22(building-building) was significantly improved. The reason for that can be described as follows: the building class, the road class,

\begin{tabular}{|c|c|c|c|c|c|}
\hline$t_{1}-t_{2}$ & lake & building & road & $\begin{array}{l}\text { farm } \\
\text { land }\end{array}$ & $\begin{array}{l}\text { bare } \\
\text { land }\end{array}$ \\
\hline lake & 0.25 & 0.12 & 0.22 & 0.16 & 0.25 \\
\hline building & 0.00 & 0.91 & 0.07 & 0.02 & 0.00 \\
\hline road & 0.03 & 0.09 & 0.83 & 0.06 & 0.00 \\
\hline farmland & 0.00 & 0.01 & 0.09 & 0.84 & 0.06 \\
\hline
\end{tabular}

Table 1.Land-cover class transition matrix

\begin{tabular}{|c|c|c|c|c|}
\hline class adjacent class & lake & building & road & $\begin{array}{l}\text { farm } \\
\text { land }\end{array}$ \\
\hline lake & 0.72 & 0.18 & 0.02 & 0.08 \\
\hline building & 0.33 & 0.52 & 0.09 & 0.06 \\
\hline road & 0.17 & 0.68 & 0.13 & 0.02 \\
\hline farmland & 0.37 & 0.15 & 0.01 & 0.47 \\
\hline
\end{tabular}

Table 2.Land-cover class adjacency matrix and the bare land class all have strong reflectivity with high gray value, thus, a classifier only considering spectral information can't distinguish them. Nonetheless, the probability of the lake at $t_{1}$ converting to bare land at $t_{2}$ is high and the building is more likely adjacent to the building than the road in space.The proposed approach considering the temporal dependence information and the spatial contextual information can distinguish the above three classes and get an improved accuracy.

\begin{tabular}{|c|c|c|c|c|c|c|c|c|c|c|c|c|c|c|c|c|c|c|c|c|}
\hline & 11 & 12 & 13 & 14 & 15 & 21 & 22 & 23 & 24 & 25 & 31 & 32 & 33 & 34 & 35 & 41 & 42 & 43 & 44 & 45 \\
\hline 11 & 65 & 1 & 0 & 4 & 1 & 14 & 0 & 0 & 0 & 0 & 3 & 0 & 0 & 0 & 0 & 5 & 0 & 0 & 1 & 0 \\
\hline 12 & 1 & 22 & 3 & 0 & 0 & 0 & 3 & 0 & 2 & 3 & 0 & 0 & 0 & 0 & 0 & 0 & 0 & 0 & 1 & 0 \\
\hline 13 & 0 & 1 & 10 & 0 & 6 & 0 & 0 & 5 & 0 & 0 & 0 & 0 & 0 & 0 & 0 & 0 & 1 & 9 & 0 & 2 \\
\hline 14 & 4 & 2 & 0 & 37 & 0 & 1 & 1 & 0 & 3 & 0 & 0 & 0 & 0 & 0 & 0 & 0 & 1 & 0 & 1 & 0 \\
\hline 15 & 1 & 19 & 49 & 12 & 56 & 0 & 3 & 9 & 1 & 9 & 0 & 0 & 0 & 0 & 0 & 0 & 0 & 3 & 0 & 9 \\
\hline 21 & 0 & 0 & 0 & 0 & 0 & 1 & 0 & 0 & 0 & 0 & 0 & 0 & 0 & 0 & 0 & 0 & 0 & 0 & 0 & 0 \\
\hline 22 & 2 & 20 & 0 & 2 & 0 & 1 & 52 & 14 & 9 & 11 & 0 & 10 & 0 & 1 & 1 & 1 & 3 & 0 & 1 & 0 \\
\hline 31 & 0 & 0 & 0 & 0 & 0 & 0 & 0 & 0 & 0 & 0 & 1 & 0 & 0 & 0 & 0 & 0 & 0 & 0 & 0 & 0 \\
\hline 32 & 0 & 0 & 0 & 0 & 0 & 0 & 1 & 1 & 0 & 1 & 0 & 1 & 0 & 0 & 1 & 0 & 0 & 0 & 0 & 0 \\
\hline 33 & 0 & 0 & 1 & 0 & 0 & 0 & 0 & 7 & 3 & 0 & 0 & 1 & 15 & 1 & 0 & 0 & 0 & 0 & 0 & 0 \\
\hline 34 & 0 & 0 & 0 & 0 & 0 & 0 & 0 & 0 & 1 & 0 & 0 & 0 & 0 & 0 & 0 & 0 & 0 & 0 & 0 & 0 \\
\hline 35 & 0 & 0 & 0 & 0 & 0 & 0 & 0 & 1 & 0 & 0 & 0 & 0 & 0 & 0 & 0 & 0 & 0 & 0 & 0 & 0 \\
\hline 41 & 0 & 1 & 0 & 1 & 0 & 1 & 0 & 0 & 0 & 0 & 0 & 0 & 0 & 0 & 0 & 0 & 0 & 0 & 0 & 0 \\
\hline 42 & 0 & 0 & 0 & 0 & 1 & 0 & 2 & 3 & 0 & 0 & 0 & 0 & 0 & 0 & 0 & 0 & 4 & 1 & 0 & 1 \\
\hline 43 & 0 & 0 & 0 & 0 & 0 & 0 & 0 & 1 & 0 & 0 & 0 & 0 & 0 & 0 & 0 & 0 & 0 & 0 & 0 & 0 \\
\hline 44 & 6 & 1 & 0 & 10 & 0 & 1 & 1 & 0 & 3 & 0 & 0 & 0 & 0 & 0 & 0 & 10 & 1 & 0 & 24 & 2 \\
\hline 45 & 0 & 0 & 0 & 0 & 0 & 0 & 0 & 0 & 0 & 0 & 0 & 0 & 0 & 0 & 0 & 0 & 0 & 0 & 0 & 1 \\
\hline
\end{tabular}

Table 3.Error matrix of the land-cover transitions of the objects by pcc algorithm 


\begin{tabular}{lllllll|l|l|l|l|l|l|l|l|l|l|l|l|l|l|l}
\hline & $\mathbf{1 1}$ & $\mathbf{1 2}$ & $\mathbf{1 3}$ & $\mathbf{1 4}$ & $\mathbf{1 5}$ & $\mathbf{2 1}$ & $\mathbf{2 2}$ & $\mathbf{2 3}$ & $\mathbf{2 4}$ & $\mathbf{2 5}$ & $\mathbf{3 1}$ & $\mathbf{3 2}$ & $\mathbf{3 3}$ & $\mathbf{3 4}$ & $\mathbf{3 5}$ & $\mathbf{4 1}$ & $\mathbf{4 2}$ & $\mathbf{4 3}$ & $\mathbf{4 4}$ & $\mathbf{4 5}$ \\
\hline $\mathbf{1 1}$ & 89 & 0 & 0 & 2 & 0 & 0 & 0 & 0 & 0 & 0 & 0 & 0 & 0 & 0 & 0 & 0 & 0 & 0 & 3 & 0 \\
\hline $\mathbf{1 2}$ & 0 & 20 & 2 & 0 & 5 & 0 & 7 & 0 & 0 & 0 & 0 & 0 & 0 & 0 & 0 & 0 & 0 & 0 & 1 & 0 \\
\hline $\mathbf{1 3}$ & 0 & 0 & 33 & 0 & 1 & 0 & 0 & 0 & 0 & 0 & 0 & 0 & 0 & 0 & 0 & 0 & 0 & 0 & 0 & 0 \\
\hline $\mathbf{1 4}$ & 2 & 0 & 0 & 47 & 1 & 0 & 0 & 0 & 0 & 0 & 0 & 0 & 0 & 0 & 0 & 0 & 0 & 0 & 0 & 0 \\
\hline $\mathbf{1 5}$ & 0 & 0 & 5 & 0 & 166 & 0 & 0 & 0 & 0 & 0 & 0 & 0 & 0 & 0 & 0 & 0 & 0 & 0 & 0 & 0 \\
\hline $\mathbf{2 1}$ & 0 & 0 & 0 & 0 & 0 & 1 & 0 & 0 & 0 & 0 & 0 & 0 & 0 & 0 & 0 & 0 & 0 & 0 & 0 & 0 \\
\hline $\mathbf{2 2}$ & 0 & 1 & 1 & 2 & 0 & 0 & 120 & 0 & 0 & 0 & 0 & 0 & 1 & 0 & 0 & 0 & 0 & 0 & 3 & 0 \\
\hline $\mathbf{3 1}$ & 0 & 0 & 0 & 0 & 0 & 0 & 0 & 0 & 0 & 0 & 1 & 0 & 0 & 0 & 0 & 0 & 0 & 0 & 0 & 0 \\
\hline $\mathbf{3 2}$ & 0 & 0 & 0 & 0 & 0 & 0 & 4 & 0 & 0 & 0 & 0 & 1 & 0 & 0 & 0 & 0 & 0 & 0 & 0 & 0 \\
\hline $\mathbf{3 3}$ & 0 & 0 & 3 & 0 & 0 & 0 & 4 & 0 & 0 & 0 & 0 & 0 & 20 & 0 & 0 & 0 & 0 & 0 & 1 & 0 \\
\hline $\mathbf{3 4}$ & 0 & 0 & 0 & 0 & 0 & 0 & 0 & 0 & 0 & 0 & 0 & 0 & 0 & 0 & 0 & 0 & 0 & 0 & 1 & 0 \\
\hline $\mathbf{3 5}$ & 0 & 0 & 0 & 0 & 1 & 0 & 0 & 0 & 0 & 0 & 0 & 0 & 0 & 0 & 0 & 0 & 0 & 0 & 0 & 0 \\
\hline $\mathbf{4 1}$ & 0 & 0 & 0 & 0 & 0 & 0 & 1 & 0 & 0 & 0 & 0 & 0 & 0 & 0 & 0 & 0 & 0 & 0 & 2 & 0 \\
\hline $\mathbf{4 2}$ & 0 & 0 & 2 & 0 & 1 & 0 & 5 & 0 & 0 & 0 & 0 & 0 & 0 & 0 & 0 & 0 & 0 & 0 & 4 & 0 \\
\hline $\mathbf{4 3}$ & 0 & 0 & 1 & 0 & 0 & 0 & 0 & 0 & 0 & 0 & 0 & 0 & 0 & 0 & 0 & 0 & 0 & 0 & 0 & 0 \\
\hline $\mathbf{4 4}$ & 0 & 0 & 0 & 4 & 0 & 0 & 0 & 0 & 0 & 0 & 0 & 0 & 0 & 0 & 0 & 0 & 0 & 0 & 55 & 0 \\
\hline $\mathbf{4 5}$ & 0 & 0 & 0 & 0 & 0 & 0 & 0 & 0 & 0 & 0 & 0 & 0 & 0 & 0 & 0 & 0 & 0 & 0 & 1 & 0 \\
\hline
\end{tabular}

Table 4.Error matrix of the land-cover transitions of the objects by the proposed algorithm

Table 5 shows the accuracy of each classification map for the single time. At both times, the proposed algorithm can provide better accuracy.

\begin{tabular}{c|c|c}
\hline \multirow{2}{*}{ Algorithm } & \multicolumn{2}{|c}{ Overall Classification Accuracy(\%) } \\
\cline { 2 - 3 } & 2002 & 2005 \\
\hline PCC & 0.71 & 0.66 \\
\hline Proposed method & 0.93 & 0.93 \\
\hline
\end{tabular}

Table 5. Accuracies of the classification maps at each time by pcc and proposed algorithm

\subsection{Change detection results}

In order to obtain the accuracy of PCC technique and proposed method, a standard change map according to the ground truth was made [Figure 5(c)]. Compared with the change detection results by PCC technique [Figure 5(a)], the proposed method [ Figure 5(b)] can provide better accuracy.

We also estimated the accuracy of the two methods by false alarms and missed alarms in Table 6. Compared with the PCC technique, the proposed method can reduce the false alarms dramatically. But the proposed method increased the missed alarms to 28. However, it can provide better overall accuracies which was reduced from 160 to 41 respectively.

\begin{tabular}{c|c|c|c}
\hline Algorithm & $\begin{array}{c}\text { False } \\
\text { alarms }\end{array}$ & $\begin{array}{c}\text { Missed } \\
\text { alarms }\end{array}$ & $\begin{array}{c}\text { Overall } \\
\text { Error }\end{array}$ \\
\hline PCC & 142 & 18 & 160 \\
\hline Proposed method & 13 & 28 & 41 \\
\hline
\end{tabular}

Table 6. Change detection results by the two methods

\section{CONCLUSIONS}

An object-oriented change detection approach considering spatiotemporal relationship in multi-temporal remote-sensing images has been proposed in this

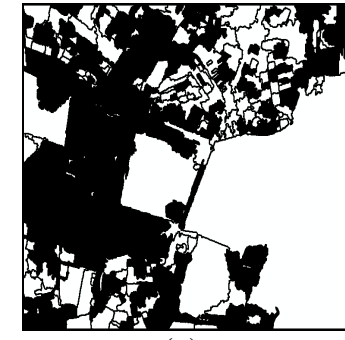

(a)

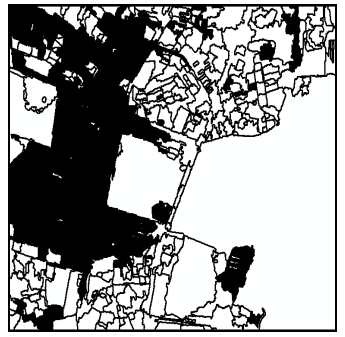

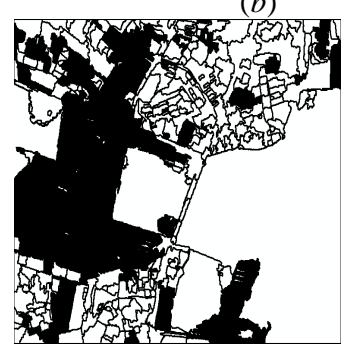

(c)

Figure 5. Change detection results(black areas denote the changed areas): (a) PCC technique with 440 changed objects and 185 unchanged objects;(b) proposed method with 301 changed objects and 324 unchanged objects; and (c) ground truth with 316 changed objects and 309 unchanged objects.

paper. The approach adopts the marker-based watershed algorithm to obtain objects. Change detection technique based on object can effectively suppress the salt and pepper noise in remote sensing imagery. The object-oriented compound classification which takes into account the spatiotemporal relationship between the two times is presented. The probabilities of land-cover class transitions are employed to describe the temporal dependence information and the Markov Random Field (MRF) model is utilized to represent the spatial-contextual 
information. Land-cover class transition matrix and adjacency matrix are computed through the compound classification results at the two times. ICM technique is adopted to get the optimal compound classification results. Change detection is achieved by comparing classification results between the two times.

The method proposed in this paper is a supervised technique. A training set is acquired by each of the multitemporal images. Thus, this method is not sensitive to atmospheric conditions and sensor calibration.

Experimental results on the QuickBird imagery show the effectiveness of the proposed method. The accuracies of the classification maps are increased. Thus the overall error of change detection is reduced. Compared with the PCC method, the proposed method can provide better accuracy for change detection.

As future developments of this paper, we plan to do as follows: 1) further analysis of the two assumptions and 2) introduce the texture information and shape information of objects into the proposed method.

\section{ACKNOWLEDGMENT}

Supported by the Geographic National Condition Monitoring Engineering Research Center of Sichuan Province (GC201506) and the science and technology plan of sichuan bureau of surveying, mapping and geo information(J2014ZC12, J2014ZC16)

\section{REFERENCE}

[1] Singh A,1989.Digital change detection techniques using remotely sensed data. Int.J.Remote Sensing,10(6),pp.989 $-1003$.

[2] Teng S P, Chen Y K , and Cheng K S, 2008. Hypothesistest-based land cover change detection using multitemporal satellite images-A comparative study. Advances in Space Research,41,pp.1744-1754.

[3] Bruzzone L, Fernàndez P D, 2000.Automatic analysis of the difference image for unsupervised change detection. IEEE Trans.Geosci.Remote Sensing, 38, pp.1171-1182.

[4] Bruzzone L, Fernàndez P D, 2002. An adaptive semipara metric and context-Based approach to unsupervised change detection in multitemporal remote-sensing images.IEEE Transaction on image processing,11(4), pp. 452-466.

[5] Fung T, 1990.An assessment of TM imagery for land -cover change detection. IEEE Trans. Geosci. Remote Sensing, 28(12), pp. 681-684.

[6] Malila,William A,1980.Change vector analysis:an approach for detecting forest changes with landsat. Machine Processing of Remotely Sensed Data Symposium,pp.326-335.

[7] Chen J, Chen X H, Cui X H,and Chen J,2011.Change vector analysis in posterior probability space: $a$ new method for land cover change detection. IEEE Geoscience and Remote Sensing Letters, 8(2), pp. 317- 321.

[8] He C Y, Wei A N,and Shi P J,et al,2011.Detecting land-use/ land-cover change in rural-urban fringe areas using extended change-vector analysis.International Journal of Applied Earth Observation and Geo- information, 13,pp.572-585.

[9] Bovolo F, Bruzzone L, 2007. A Theoretical Framework for Unsupervised Change Detection Based on Change Vector Analysis in the Polar Domain. IEEE Trans.Geosci. Remote Sensing, 5(1),pp.218-236,

[10]Varshney A, Arora M K and Ghosh J K,2012.Median change vector analysis algorithm for land-use land-cover change detection from remote-sensing data,Remote Sensing Letters, 3(7),pp.605-614.

[11] Bruzzone L, Serpico S B, 1997. An iterative technique for the detection of landcover transitionsin multi temporal remote-sensing images. IEEE Trans. Geosci. Remote Sensing, 35(4),pp.858-867.

[12] Bovolo F, Bruzzone L, 2009. A multilevel parcel-based approach to change detection in very high resolution multite-mporal images. IEEE Geoscience and Remote Sensing Letters,6,pp.33-37.

[13] L.Bruzzone and D.F.Prieto, "An adaptive parcel-based technique for unsupervised change detection," Int.J. Remote Sensing,vol.21, no.4,pp.817-822,Mar 2000

[14] Tang Y Q, Zhang L P, Huang X, 2011. Object-oritented change detection based on the Kolmogorov-Simirnov test using high-resolution multispectral imagery .Int.J. Remote Sensing, 32(20),pp.5719-5740.

[15] Im J, Jensen J R,and Tullis J A,2008.Object-based change detection using correlation image analysis and image segmentation. Int.J.Remote Sensing,29(2), pp. 399-423.

[16] Walter V, 2004. Object-based classification of remote sensing data for change detection.ISPRS Journal of Photogrammetry and Remote Sensing,58,pp.225-238,

[17] Gao H, Siu W C, Hou C H, 2001. Improved techniques for automatic image segmentation.IEEE Transactions on Circuits and Systems for Video Technology, 11(12), pp. 1273-1280,

[18] Salembier P, Pardas M,1994. Hierarchica morphological segmentation for image sequence coding. IEEE Transaactions on Image Processing,3(5),pp.639-651.

[19] Li D R, Zhang G F, Wu Z C, and et al, 2010. An edge embedded marker-based watershed algorithm for high spatial resolution remote sensing image segmentation. IEEE Transactions on Image Processing,19(10), pp.50 $-57$.

[20] Paul R H, Canagarajah C N, David R B, 2003. Image segmentation using a texture gradient based watershed transform.IEEE Transactions on Image Processing 12 (12),pp.1618-1633.

[21] Haris K, Fstratiadis S N E, Maglaveras N, and et al, 1998. Hybrid image segmentation using watersheds and fast region merging.IEEE Trans on image processing,7 (12), pp.1684-1699.

[22] Meyer F, 1992. Color Image Segmentation. IEEE International Conference on Image Processing and Its Applications, Maastricht, Netherlands.

[23] Gao H, .Lin W S, Xue P.and Siu W C, 2006. Markerbased image segmentation relying on disjoint set union. Signal Processing: Image Communication, 21,pp.100 112.

[24] Dubes R C,Jain A K,1989.Random field models in image analysis. Journal of applied statistics, 16, pp.131 -163 .

[25] Besag J, 1986. On the statistical analysis of dirty pictures. J. Royal Statist.Soc.B.48,pp.259-302. 